This item was submitted to Loughborough's Research Repository by the author.

Items in Figshare are protected by copyright, with all rights reserved, unless otherwise indicated.

\title{
A Galerkin-type state-space approach for transverse vibrations of slender double-beam systems with viscoelastic inner layer
}

PLEASE CITE THE PUBLISHED VERSION

http://www.sciencedirect.com/science/article/pii/S0022460X11006183

PUBLISHER

(C) Elsevier

VERSION

AM (Accepted Manuscript)

LICENCE

CC BY-NC-ND 4.0

\section{REPOSITORY RECORD}

Palmeri, Alessandro, and Sondipon Adhikari. 2019. "A Galerkin-type State-space Approach for Transverse Vibrations of Slender Double-beam Systems with Viscoelastic Inner Layer”. figshare.

https://hdl.handle.net/2134/8373. 
This item was submitted to Loughborough's Institutional Repository (https://dspace.lboro.ac.uk/) by the author and is made available under the following Creative Commons Licence conditions.

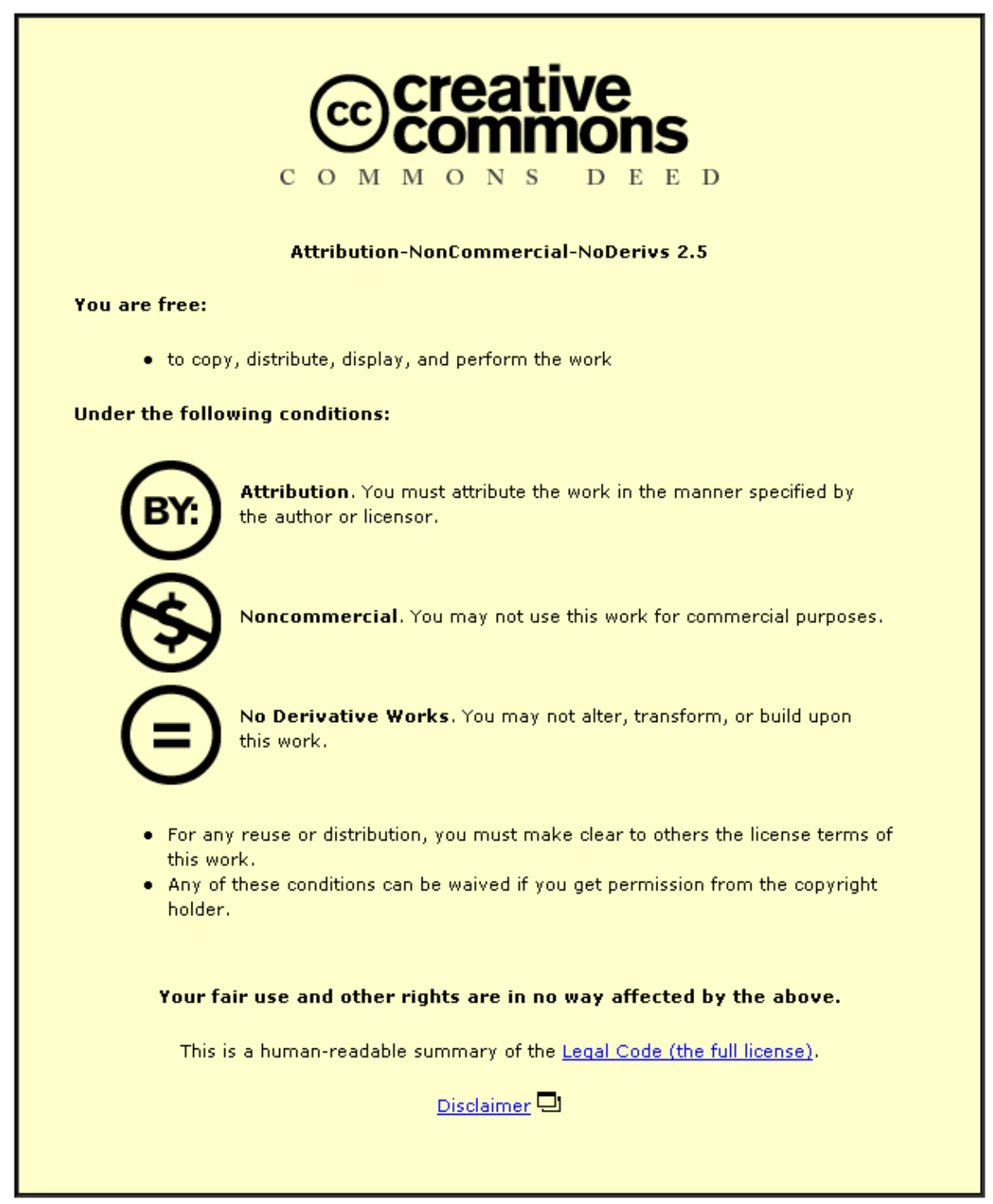

For the full text of this licence, please go to: http://creativecommons.org/licenses/by-nc-nd/2.5/ 


\title{
A Galerkin-type state-space approach for transverse vibrations of slender double-beam systems with viscoelastic inner layer
}

\author{
Alessandro Palmeri ${ }^{\mathrm{a}, *}$, Sondipon Adhikari ${ }^{\mathrm{b}}$ \\ ${ }^{a}$ Department of Civil and Building Engineering, Loughborough University, Sir Frank \\ Gibb Building, Loughborough LE11 3TU, United Kingdom \\ ${ }^{b}$ College of Engineering, Swansea University, Singleton Park, Swansea SA2 8PP, United \\ Kingdom
}

\begin{abstract}
A novel state-space form for studying transverse vibrations of doublebeam systems, made of two outer elastic beams continuously joined by an inner viscoelastic layer, is presented and numerically validated. As apposite to other methods available in the literature, the proposed technique enables one to considerer $i$ ) inhomogeneous systems, $i i$ ) any boundary conditions and iii) rate-dependent constitutive law for the inner layer. The formulation is developed by means of Galerkin-type approximations for the fields of transverse displacements in the system. Numerical examples demonstrate that the proposed approach is accurate and versatile, and lends itself to be used for both frequency- and time-domain analyses.

Keywords: Euler-Bernoulli beams, Sandwich beams, Standard Linear Solid (SLS) model, State-space formalism, Viscoelastic damping
\end{abstract}

\footnotetext{
${ }^{*}$ Corresponding Author

Email addresses: A.Palmeri@lboro.ac.uk, Dynamics.Structures@gmail.com (Alessandro Palmeri ), S.Adhikari@swansea.ac.uk (Sondipon Adhikari) 


\section{Introduction}

Beams are fundamental components in most of the structural systems conceived, designed and constructed in civil, mechanical and aerospace engineering. Hence, free and forced vibrations of single Euler-Bernoulli and Timoshenko beams are covered in hundreds of scientific and technical contributions. On the other hand, relatively few papers have been published on the dynamics of double-beam systems, made of two parallel slender beams continuously connected by a Winkler-type viscoelastic layer.

Despite analytical and numerical difficulties arising in the solution of the coupled partial differential equations governing the motion, this dynamic system is certainly worth of investigation. As an example, a double-beam model can be effective in approximating the dynamic behaviour of sandwich beams, largely used in many engineering situations $[1,2]$. Motivated by the recent development of the nano-opto-mechanical systems (NOMS) [3, 4, 5], Murmu and Adhikari $[6,7,8]$ have considered the dynamics and instability of nanoscale double-beam systems using scale-dependent non-local theory. A continuous dynamic vibration absorber (CDVA) is another important case of double-beam system, where secondary beam and inner layer are designed in order to mitigate the vibration experienced by the primary beam [9]. The double-beam model is also able to capture the dynamic response of floatingslab railway tracks, widely used to control vibration due to underground trains $[10]$.

Several interesting analytical works have been developed in recent years which demonstrate an emerging attention to this subject. $\mathrm{Vu}$ et al. [11] formulated a closed-form solution for the vibration of a viscously damped 
double-beam system subjected to harmonic excitations. Two restrictions, however, limit the practical applicability of this solution: i) outer beams must be homogenous and identical; ii) boundary conditions on the same side of the system must be the same. Oniszczuk [12, 13] presented some analytical expressions for the undamped free and forced vibrations of a simplysupported double-beam system. In his formulation the outer beams can be different from each other, but they must be homogeneous and pinned at the ends; moreover, the damping is totally neglected. Abu-Hilal [14], under the same assumptions as in Ref. [11], studied the dynamic response of a double-beam system traversed by a moving force.

Several authors have considered distributed parameter systems with viscoelastic damping. In one of the earliest work Banks and Inman [15] have considered viscoelastically damped beam. They have taken four different models of damping: viscous air damping, Kelvin-Voigt damping, time hysteresis damping and spatial hysteresis damping, and used a spline inverse procedure to form a least-square fit to the experimental data. Cortes and Elejabarrieta $[16,17]$ considered free and forced vibration analysis of axially vibrating rod with viscoelastic damping. Chen [18] considered bending vibration of axially loaded Timoshenko beams with locally distributed KelvinVoigt type of damping. Yadav [19] considered the dynamics of a four-layer beam with alternate elastic layer and viscoelastic layer.

The effects of a viscoelastic inner layer on the dynamics of double-beam systems have been addressed by Palmeri and Muscolino [20] by using a component-mode synthesis (CMS) approach, whose practical applicability is limited by the need to solve a fourth-order differential equation for each 
assumed mode; moreover, the effects of inner transverse vibrations within the viscoelastic core is neglected. In this paper, aimed at overcoming the severe limitations highlighted above, a novel Galerkin-type state-space model for the vibration analysis of double-beam systems is formulated and numerically validated. Based on a convenient choice of assumed modes for the components, the proposed technique allows us to considerer inhomogeneous beams and any boundary conditions. Furthermore, since in many engineering applications an elastomeric material is used in the inner layer, the latter is described through the so-called Standard Linear Solid (SLS) model, which is one of the simplest rheological models able to represent the rate-dependent behaviour of viscoelastic solids [21]. The effects of the viscoelastic damping on the double-beam system is represented by generalizing the concept of modal relaxation function, recently suggested by Palmeri and his associates $[22,23]$.

It is worth noting that, being based on the introduction of a set of additional internal variables, the extension of the proposed technique to more refined rheological models, such as the generalized Maxwell's model or the Laguerre polynomial approximation [24], is quite straightforward. It is also worth mentioning that several works exist in the frequency [25, 26, 27, 28, 29] and also in the time domain [30, 31, 32, 33] for the generalized Maxwell's model in the context of discrete systems, while the proposed approach is specifically tailored to continuous structures, which have received less attention in the past $[23,34]$. 


\section{Statement of the problem}

\subsection{Basic assumptions}

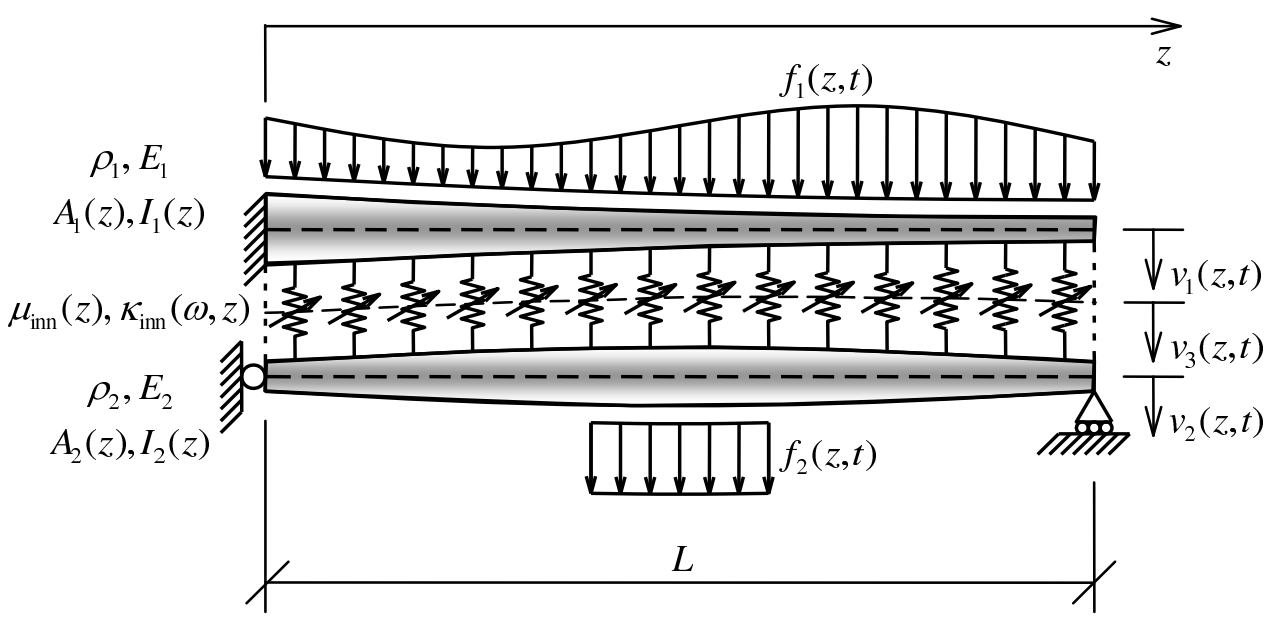

(a)

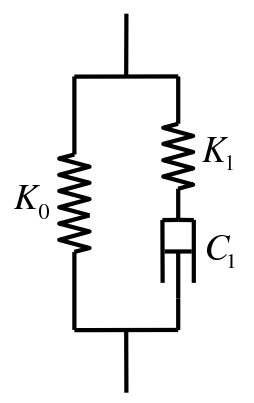

(b)

Figure 1: Double-beam dynamic system (a); Standard Linear Solid (SLS) rheological model (b)

The dynamic system under investigation is made of two parallel elastic beams of the same length $L$, subjected to arbitrary time-dependent transverse forces and continuously joined by an inner layer of Winkler-type viscoelastic sprigs (Fig. 1a). Both outer beams are assumed to be slender, and therefore 
the classical Euler-Bernoulli beam theory is adopted in deriving the equations of motion, i.e. the effects of both rotational inertia and shear strain are neglected in this study.

In general, the two outer beams have different mechanical properties and are inhomogeneous: thus, they are fully characterized by modulus of elasticity $E_{r}$, mass density $\rho_{r}$, cross-sectional area $A_{r}(z)$ and second moment $I_{r}(z)$, where the subscript $r=1,2$ denotes first (top) and second (bottom) beam, respectively, while the variable $z \in[0, L]$ is the abscissa along the beams. Moreover, the inherent damping of the outer beams is described by the frequency-independent viscous damping ratios $\zeta_{r}$.

The inner layer too is allowed to be inhomogeneous: therefore, it is fully characterized by mass per unit length, $\mu_{\mathrm{inn}}(z)$, which depends on the spatial coordinate $z$ only, and complex-valued stiffness per unit length, $\kappa_{\text {inn }}(\omega, z)$, which depends also on the vibration frequency $\omega$. In the following, these functions are conveniently expressed as $\mu_{\mathrm{inn}}(z)=\bar{m}_{\mathrm{inn}} \alpha_{\mathrm{M}}(z)$ and $\kappa_{\mathrm{inn}}(\omega, z)=$ $\bar{k}_{\text {inn }}(\omega) \alpha_{\mathrm{K}}(z)$, respectively, where $\bar{m}_{\text {inn }}$ and $\bar{k}_{\text {inn }}(\omega)$ are the corresponding reference quantities, e.g. at the position where $z=0$ or $z=L / 2$, while $\alpha_{\mathrm{M}}(z)$ and $\alpha_{\mathrm{K}}(z)$ are two dimensionless functions of the abscissa $z$.

\subsection{Viscoelastic model of the inner layer}

For the sake of simplicity, the dynamic behaviour of the viscoelastic inner layer is described in our formulation by the Standard Linear Solid (SLS) model (Fig. 1b), which is made of a primary elastic spring (equilibrium modulus), $K_{0} \equiv \bar{k}_{\text {inn }}(0)$, in parallel with a Maxwell's element, given by a secondary elastic spring, $K_{1}$, in series with a viscous dashpot, $C_{1}=K_{1} \tau_{1}, \tau_{1}$ being the so-called relaxation time of the viscoelastic material. The reference 
complex-valued stiffness $\bar{k}_{\text {inn }}(\omega)$, thus, takes the expression:

$$
\bar{k}_{\text {inn }}(\omega)=K_{0}+K_{1} \frac{\imath \tau_{1} \omega}{1+\imath \tau_{1} \omega}
$$

where $\imath=\sqrt{-1}$ is the imaginary unit. In a mixed time-frequency domain, the reaction force, $F(t)$, experienced by the SLS model can be related to the pertinent displacement, $\delta(t)$, as:

$$
F(t)=\bar{k}_{\text {inn }}(\omega) \delta(t)
$$

Although not formally rigorous, Eq. (2) has the merit to highlight the dependence on the vibration frequency of the reaction force. As an alternative, the force-displacement relationship can be rigorously expressed in the time domain as [21, 24]:

$$
F(t)=\bar{\varphi}_{\mathrm{inn}}(t) * \dot{\delta}(t)=\int_{-\infty}^{t} \bar{\varphi}_{\mathrm{inn}}(t-s) \dot{\delta}(s) \mathrm{d} s
$$

where the asterisk $*$ stands for the convolution operator, the over-dot means derivative with respect to time $t$, so that $\dot{\delta}(t)$ is the pertinent velocity, while $\bar{\varphi}_{\text {inn }}(t)$ is the relaxation function of the SLS model, given by:

$$
\bar{\varphi}_{\text {inn }}(t)=\mathcal{F}^{-1}\left\langle\frac{1}{\imath \omega} \bar{k}_{\text {inn }}(\omega)\right\rangle=\left(K_{0}+K_{1} \mathrm{e}^{-t / \tau_{1}}\right) \mathcal{U}(t),
$$

in which $\mathcal{F}^{-1}$ is the inverse Fourier transform operator, while $\mathcal{U}$ is the Heaviside unit step function continuous from the right, i.e. $\mathcal{U}(t)=0$ when $t<0$, and $\mathcal{U}(t)=1$ when $t \geq 0$. In Ref. [24] it is demonstrated that the reaction force $F(t)$ can be also expressed as:

$$
F(t)=K_{0} \delta(t)+K_{1} \lambda_{1}(t)
$$


where $K_{0} \delta(t)$ is the mere elastic part in the viscoelastic constitutive law, while $K_{1} \lambda_{1}(t)$ is the contribution of the Maxwell's element, $\lambda_{1}(t)$ being an additional internal variable, which in turn measures the elongation of the spring $K_{1}$ and is ruled by:

$$
\dot{\lambda}_{1}(t)=\dot{\delta}(t)-\frac{\lambda_{1}(t)}{\tau_{1}}
$$

\section{Undamped vibrations}

Let us considerer initially a double-beam system which does not possess any damping mechanism, i.e. the limiting situation where both the viscous damping ratios $\zeta_{1}$ and $\zeta_{2}$ of the outer beams are assumed to be zero and the viscous coefficient $C_{1}$ of the inner viscoelastic layer goes to zero too. It is worth noting that in this case the core becomes purely elastic, as considered in Refs. $[12,13]$; in contrast with these studies, however, in our formulation the outer beams can be inhomogeneous and with any boundary conditions.

\subsection{Assumed modes}

For the $r$ th beam, individually considered, a convenient array of shape functions (or assumed modes) can be defined by taking the first $n$ buckling modes of the homogenized beam, $\phi_{r}(z)=\left\{\phi_{r, 1}(z) \cdots \phi_{r, n}(z)\right\}^{\top}$, the superscripted symbol $\mathrm{T}$ denoting the transpose operator. These shape functions, thus, are solution of the classical eigenproblem:

$$
\phi_{r, j}^{\prime \prime \prime \prime}(z)+\alpha_{r, j}^{2} \phi_{r, j}^{\prime \prime}(z)=0
$$

where the prime denotes derivative with respect to the spatial coordinate $z$, while $\left\{\phi_{r, j}(z), \alpha_{r, j}\right\}$ is the $j$ th pair of eigenfunction and eigenvalue for the 
Table 1: Assumed modes for the outer beams (see e.g. Ref. [35])

\begin{tabular}{c|cc}
$\begin{array}{c}\text { Boundary } \\
\text { conditions }\end{array}$ & $\begin{array}{c}\text { Eigenfunctions } \\
\phi_{r, j}(z)\end{array}$ & $\begin{array}{c}\text { Eigenvalue } \\
\text { equation }\end{array}$ \\
\hline $\mathrm{P}-\mathrm{P}$ & $\sin \left(\alpha_{r, j} z\right)$ & $\alpha_{r, j}=\frac{j \pi}{L}$ \\
$\mathrm{C}-\mathrm{F}$ & $1-\cos \left(\alpha_{r, j} z\right)$ & $\alpha_{r, j}=\frac{(2 j-1) \pi}{2 L}$ \\
$\mathrm{C}-\mathrm{P}$ & $\cos \left(\alpha_{r, j} z\right)-\frac{\sin \left(\alpha_{r, j} z\right)}{\alpha_{r, j} L}+\frac{z-L}{L}$ & $\tan \left(\alpha_{r, j} L\right)=\alpha_{r, j} L$ \\
$\mathrm{C}-\mathrm{C}$ & $\cos \left(\alpha_{r, j} z\right)-1$ & $\alpha_{r, j}=\frac{2 j \pi}{L}$ \\
\hline
\end{tabular}

$r$ th beam. The non-trivial solutions satisfying Eq. (7) are offered in Tab. 1 for different boundary conditions of the $r$ th beam at $z=0$ and $z=L$, e.g. Pinned-Pinned (P-P), Clamped-Free (C-F), Clamped-Pinned (C-P) and Clamped-Clamped (C-C).

If the $r$ th beam is kinematically unstable when considered individually (i.e. when the restraining due to the other beam is neglected), e.g. if the boundary conditions for the $r$ th beam are Pinned-Free $(\mathrm{P}-\mathrm{F})$ or Free-Free $(\mathrm{F}-\mathrm{F})$, the shape functions $\phi_{r, j}(z)$ assumed in our study are those of the $\mathrm{P}-\mathrm{P}$ beam, complemented by one $(\mathrm{P}-\mathrm{F})$ or two $(\mathrm{F}-\mathrm{F})$ rigid-body functions, as shown in Tab. 2.

Once the arrays $\phi_{r}(z)$ are defined for top $(r=1)$ and bottom $(r=2)$ beams, the time-varying field $v_{r}(z, t)$ of transverse displacements in the $r$ th outer beam can be expressed as:

$$
v_{r}(z, t)=\phi_{r}^{\top}(z) \cdot \mathbf{q}_{r}(t)=\sum_{j=1}^{n} \phi_{r, j}(z) q_{r, j}(t),
$$

in which the dot - denotes matrix product, while the $n$-dimensional array 
Table 2: Additional rigid-body modes for kinematically unstable beams

\begin{tabular}{c|c}
$\begin{array}{c}\text { Boundary } \\
\text { conditions }\end{array}$ & $\begin{array}{c}\text { Further } \\
\text { assumed modes }\end{array}$ \\
\hline $\mathrm{P}-\mathrm{F}$ & $\phi_{i, n}(z)=z / L$ \\
$\mathrm{~F}-\mathrm{F}$ & $\phi_{i, n-1}(z)=1 ; \quad \phi_{i, n}(z)=(2 z-L) / L$ \\
\hline
\end{tabular}

$\mathbf{q}_{r}(t)=\left\{q_{r, 1}(t) \cdots q_{r, n}(t)\right\}^{\top}$ collects the Lagrangian coordinates associated with the assumed modes for the $r$ th beam.

Analogously, the time-varying field of transverse displacements at the intermediate position of the inner layer can be represented as:

$$
v_{3}(z, t)=\phi_{3}^{\top}(z) \cdot \mathbf{q}_{3}(t)=\sum_{j=1}^{n} \phi_{3, j}(z) q_{3, j}(t),
$$

in which the $n$-dimensional array $\phi_{3}(z)$ collects the assumed modes for F$\mathrm{F}$ boundary conditions, while $\mathbf{q}_{3}(t)$ is the associated array of Lagrangian coordinates. This additional field $v_{3}(z, t)$ enables us to take into account the transverse vibrations of the inner layer, whose mass can be conveniently lumped at top $(r=1)$, bottom $(r=2)$ and central $(r=3)$ positions of the core (see Fig. 1a). The representation of transverse displacements within the inner layer can be further improved by discretising the core with more internal points and introducing more internal fields $v_{4}(z, t), v_{5}(z, t), \cdots$ (this could be useful, for instance, to study the propagation of elastic waves orthogonally to the axes of the outer beams). This refinement is outside of the scope of present investigations. It is worth noting that, as an alternative [20], inner displacements' field can be expressed as $v_{3}(z, t)=\frac{1}{2}\left[v_{1}(z, t)+v_{2}(z, t)\right]$, 
which reduces the computational order (i.e. the size of the matrices $\mathbf{M}$ and $\mathbf{K}$ introduced below becomes smaller), but does not provide accurate information about the high-frequency dynamics of the inner layer.

According to Eqs. (8) and (9), which fully define the approximate kinematics of the double-beam system under analysis, total kinetic energy, $T(t)$, and total potential energy, $V(t)$, can be now evaluated as the sum of three terms:

$$
\begin{aligned}
& T(t)=T_{1}(t)+T_{2}(t)+T_{3}(t) ; \\
& V(t)=V_{1}(t)+V_{2}(t)+V_{3}(t) .
\end{aligned}
$$

For the outer beams $(r=1,2)$, the expressions of kinetic energy and potential energy are given by:

$$
\begin{gathered}
T_{r}(t)=\frac{1}{2} \int_{0}^{L} \mu_{r}(z)\left[\dot{v}_{r}(z, t)\right]^{2} \mathrm{~d} z ; \\
V_{r}(t)=\frac{1}{2} E_{r} \int_{0}^{L} I_{r}(z)\left[v_{r}^{\prime \prime}(z, t)\right]^{2} \mathrm{~d} z,
\end{gathered}
$$

while the contributions of the inner layer take the form:

$$
\begin{aligned}
T_{3}(t)=\frac{1}{4} \bar{m}_{\text {inn }} \int_{0}^{L} \alpha_{\mathrm{M}}(z)\left[\dot{v}_{3}(z, t)\right]^{2} \mathrm{~d} z & \\
V_{3}(t)=K_{0} \int_{0}^{L} \alpha_{\mathrm{K}}(z)\{ & {\left[v_{1}(z, t)-v_{3}(z, t)\right]^{2} } \\
+ & {\left.\left[v_{2}(z, t)-v_{3}(z, t)\right]^{2}\right\} \mathrm{d} z . }
\end{aligned}
$$

In the expressions above, $\mu_{r}(z)=\rho_{r} A_{r}(z)+\frac{1}{4} \mu_{\text {inn }}(z)$, with $r=1,2$, is the mass per unit length associated with the $r$ th outer beam, which includes the pertinent contribution of the inner layer, i.e. 1/4 of the intermediate mass, 
while the residual mass not attached to the outer beams, $\mu_{3}(z)=\frac{1}{2} \mu_{\mathrm{inn}}(z)$, is assumed to be lumped at halfway position of the inner layer.

Substituting Eqs. (8) and (9) into Eqs. (11) to (12) leads to the following expressions of kinetic energy and potential energy for the outer EulerBernoulli beams $(r=1,2)$ :

$$
\begin{aligned}
& T_{r}(t)=\frac{1}{2} \sum_{j=1}^{n} \sum_{k=1}^{n} M_{j, k}^{(r, r)} \dot{q}_{r, j}(t) \dot{q}_{r, k}(t) ; \\
& V_{r}(t)=\frac{1}{2} \sum_{j=1}^{n} \sum_{k=1}^{n} K_{j, k}^{(r, r)} q_{r, j}(t) q_{r, k}(t),
\end{aligned}
$$

and for the inner Winkler-type layer $(r=3)$ :

$$
\begin{aligned}
& T_{3}(t)=\frac{1}{2} \sum_{j=1}^{n} \sum_{k=1}^{n} M_{j, k}^{(3,3)} \dot{q}_{3, j}(t) \dot{q}_{3, k}(t) ; \\
V_{3}(t)= & \frac{1}{2} \sum_{j=1}^{n} \sum_{k=1}^{n}\left[K_{j, k}^{(3,3)} q_{3, j}(t) q_{3, k}(t)\right. \\
& +\Delta K_{j, k}^{(1,1)} q_{1, j}(t) q_{1, k}(t)+\Delta K_{j, k}^{(2,2)} q_{2, j}(t) q_{2, k}(t) \\
& \left.+K_{j, k}^{(1,3)} q_{1, j}(t) q_{3, k}(t)+K_{j, k}^{(2,3)} q_{2, j}(t) q_{3, k}(t)\right]
\end{aligned}
$$

Coefficients $M_{j, k}^{(r, r)}, K_{j, k}^{(r, s)}$ and $\Delta K_{j, k}^{(r, r)}$ in Eqs. (13) and (14) are mass and stiffness coefficients coupling the $j$ th assumed mode of the $r$ th subsystem with the $k$ th assumed mode of $r$ th $(M$ and $\Delta K)$ or $s$ th $(K)$ subsystem. The expressions of these coefficients are provided in Appendix A. It is worth emphasising here that the only coupling between the three subsystems (outer beams and inner layer) is due to the stiffness coefficients $K_{j, k}^{(1,3)}$ and $K_{j, k}^{(2,3)}$, appearing in the right-hand side of Eq. (14b). 
The generalised force $Q_{r, j}(t)$ associated with the Lagrangian coordinate $q_{r, j}(t)$ can be obtained by projecting the external dynamic loads $f_{r}(z, t)$, acting on the $r$ th layer, onto the $j$ th assumed mode for such subsystem:

$$
\begin{aligned}
Q_{r, j}(t) & =\int_{0}^{L} f_{r}(z, t) \frac{\partial}{\partial q_{r, j}(t)} v_{r}(z, t) \mathrm{d} z \\
& =\int_{0}^{L} f_{r}(z, t) \phi_{r, j}(z) \mathrm{d} z .
\end{aligned}
$$

Analogously to the array of Lagrangian coordinates $\mathbf{q}_{r}(t)$ for the $r$ th subsystem, the new $n$-dimensional forcing array $\mathbf{Q}_{r}(t)=\left\{Q_{r, 1}(t) \cdots Q_{r, n}(t)\right\}^{\top}$ can be introduced.

\subsection{Lagrangian equations of motion}

Once all the sources of kinetic and potential energies are expressed as functions of generalized displacements and velocities (Eqs. (13) and (14)), and once the generalized forces are defined (Eq. (15)), the Lagrange's equations ruling the undamped vibrations of the coupled dynamic system can be formally written as (for $r=1,2,3$ and $j=1, \cdots, n$ ):

$$
\frac{\mathrm{d}}{\mathrm{d} t}\left[\frac{\partial}{\partial \dot{q}_{r, j}(t)} \mathcal{L}(t)\right]-\frac{\partial}{\partial q_{r, j}(t)} \mathcal{L}(t)=Q_{r, j}(t),
$$

where $\mathcal{L}(t)=T(t)-V(t)$ is the so-called Lagrangian function of the system, $T(t)$ and $V(t)$ being those of Eqs. (10).

After some algebra, Eqs. (16) can be reduced to the more compact matrix form:

$$
\mathbf{M} \cdot \ddot{\mathbf{u}}(t)+\mathbf{K} \cdot \mathbf{u}(t)=\mathbf{F}(t),
$$

where the arrays $\mathbf{u}(t)$ and $\mathbf{F}(t)$, of size $3 n$, collects Lagrangian coordinates and generalised forces for the three subsystems, respectively:

$$
\mathbf{u}(t)=\left\{\mathbf{q}_{1}^{\top}(t): \mathbf{q}_{2}^{\top}(t): \mathbf{q}_{3}^{\top}(t)\right\}^{\top}
$$




$$
\mathbf{F}(t)=\left\{\mathbf{Q}_{1}^{\top}(t) \mathbf{Q}_{2}^{\top}(t): \mathbf{Q}_{3}^{\top}(t)\right\}^{\top},
$$

while $\mathbf{M}$ and $\mathbf{K}$ are the generalized mass and stiffness matrices, of dimensions $3 n \times 3 n:$

$$
\begin{aligned}
& \mathbf{M}=\left[\begin{array}{c:c:c}
\mathbf{M}^{(1,1)} & \circ & \circ \\
\hdashline \circ & \mathbf{M}^{(2,2)} & \circ \\
\hdashline 0 & \circ & \mathbf{M}^{(3,3)}
\end{array}\right] \\
& \mathbf{K}=\left[\begin{array}{c:c:c}
\mathbf{K}^{(1,1)}+\Delta \mathbf{K}^{(1,1)} & \circ & \mathbf{K}^{(1,3)} \\
\hdashline \circ & \mathbf{K}^{(2,2)}+\Delta \mathbf{K}^{(2,2)} & \mathbf{K}^{(2,3)} \\
\hdashline\left[\mathbf{K}^{(1,3)}\right]^{\mathbf{T}} & {\left[\mathbf{K}^{(2,3)}\right]^{\mathbf{\top}}} & \mathbf{K}^{(3,3)}
\end{array}\right],
\end{aligned}
$$

in which the symbol $\circ$ stands for a zero block in the mass and stiffness assemblies. It is worth emphasising here that matrix assembly procedures are not required in this case, as the mass and stiffness coefficients can be directly allocated.

Since $\mathbf{M}$ and $\mathbf{K}$ constitute a pair of real-valued symmetric matrices, they can be simultaneously diagonalised through the classical eigenproblem:

$$
\tilde{\omega}_{j}^{2} \mathbf{M} \cdot \tilde{\mathbf{x}}_{j}=\mathbf{K} \cdot \tilde{\mathbf{x}}_{j} ; \quad \tilde{\mathbf{x}}_{j}^{\top} \cdot \mathbf{M} \cdot \tilde{\mathbf{x}}_{k}=\delta_{j, k},
$$

where $\delta_{j, k}$ is the Kronecker's delta symbol, so that $\delta_{j, k}=1$ when $j=k$ and $\delta_{i, k}=0$ when $j \neq k$, and where $\tilde{\omega}_{j}$ is the approximate $j$ th natural circular frequency of the undamped double-beam system, while the corresponding approximate modal shape is given by the three-dimensional vector:

$$
\tilde{\mathbf{v}}_{j}(z)=\left\{\begin{array}{lll}
\tilde{v}_{1, j}(z) & \tilde{v}_{2, j}(z) & \tilde{v}_{3, j}(z)
\end{array}\right\}^{\top}=\boldsymbol{\Gamma}(z) \cdot \tilde{\mathbf{x}}_{j}
$$

$\boldsymbol{\Gamma}(z)$ being the $3 \times(3 n)$ transformation matrix so defined:

$$
\Gamma(z)=\left[\begin{array}{c:c:c}
\phi_{1}^{\top}(z) & \circ & \circ \\
\circ & \phi_{2}^{\top}(z) & \circ \\
\circ & \circ & \phi_{3}^{\top}(z)
\end{array}\right] .
$$


Eqs. (17) can be therefore reduced to the following modal form:

$$
\ddot{\boldsymbol{\theta}}(t)+\boldsymbol{\Omega}^{2} \cdot \boldsymbol{\theta}(t)=\mathbf{X}^{\top} \cdot \mathbf{F}(t)
$$

where $\boldsymbol{\theta}(t)=\left\{\theta_{1}(t) \cdots \theta_{m}(t)\right\}^{\top}$ is the array listing the first $m$ modal coordinates of the double-beam system under investigation, with $m \leq 3 n$, $\boldsymbol{\Omega}=\operatorname{diag}\left\{\tilde{\omega}_{1}, \cdots, \tilde{\omega}_{m}\right\}$ is the associated $m \times m$ diagonal spectral matrix,

while $\mathbf{X}=\left[\begin{array}{l:l:l}\tilde{\mathbf{x}}_{1} & \cdots & \tilde{\mathbf{x}}_{m}\end{array}\right]$, is the $n \times m$ corresponding modal matrix, whose $j$ th column is the $j$ th eigenvector $\tilde{\mathbf{x}}_{j}$ satisfying Eqs. (20).

\section{Damped vibrations}

With the aim of including energy dissipation into the equations of motions, let us generalize Eq. (17) in a convenient mixed time-frequency domain, where pure viscous damping in the outer beams and rate-dependent part of the viscoelastic constitutive law of the inner layer can be easily introduced:

$$
\mathbf{M} \cdot \ddot{\mathbf{u}}(t)+\mathbf{C} \cdot \dot{\mathbf{u}}(t)+\left[\mathbf{K}+\left(\bar{k}_{\mathrm{inn}}(\omega)-K_{0}\right) \mathbf{L}_{\mathrm{inn}}\right] \cdot \mathbf{u}(t)=\mathbf{F}(t),
$$

where $\mathbf{C}$ is the viscous damping matrix associated with energy dissipation in the outer beams, while $\mathbf{L}_{\mathrm{inn}}$ is the influence matrix of the inner layer, given by:

$$
\mathbf{L}_{\mathrm{inn}}=\frac{1}{K_{0}}\left[\begin{array}{c:c:c}
\Delta \mathbf{K}^{(1,1)} & \circ & \mathbf{K}^{(1,3)} \\
\hdashline\left[\mathbf{K}^{(1,3)}\right]^{\boldsymbol{\top}} & {\left[\mathbf{K}^{(2,3)}\right]^{\boldsymbol{\top}}} & \mathbf{K}^{(3,3)}
\end{array}\right] .
$$

For the viscous damping matrix $\mathbf{C}$, the following expression is suggested:

$$
\mathbf{C}=\left[\begin{array}{c:c:c}
\mathbf{C}^{(1,1)} & 0 & 0 \\
\hdashline 0 & \mathbf{C}^{(2,2)} & 0 \\
\hdashline 0 & 0 & 0
\end{array}\right],
$$


where the $n \times n$ block $\mathbf{C}^{(r, r)}$ is the viscous damping matrix of the $r$ th beam individually considered $(r=1,2)$. If the Rayleigh's model is adopted [36, 37], these blocks can be computed as:

$$
\mathbf{C}^{(r, r)}=2 \zeta_{r}\left[a_{\mathrm{M}} \mathbf{M}^{(r, r)}+a_{\mathrm{K}} \mathbf{K}^{(r, r)}\right]
$$

in which the coefficients $a_{\mathrm{M}}$ and $a_{\mathrm{K}}$ are given by:

$$
a_{\mathrm{M}}=\frac{\Omega_{1} \Omega_{2}}{\Omega_{1}+\Omega_{2}} ; \quad a_{\mathrm{K}}=\frac{1}{\Omega_{1}+\Omega_{2}},
$$

where the non-zero values of the circular frequencies $\Omega_{1}$ and $\Omega_{2}$ have to be properly selected. For instance, $\Omega_{1}$ can be taken as the fundamental circular frequency of the double-beam system, i.e. $\Omega_{1}=\tilde{\omega}_{1}$, while $\Omega_{2}>\Omega_{1}$ can be set among the higher circular frequencies which provide a significant contribution to the dynamic response, e.g. $\Omega_{2}=\tilde{\omega}_{m}$.

By using the same modal transformation of variables as in the previous subsection, $\mathbf{u}(t)=\mathbf{X} \cdot \boldsymbol{\theta}(t)$, Eq. (23) reduces to:

$$
\begin{aligned}
\ddot{\boldsymbol{\theta}}(t) & +\boldsymbol{\Xi} \cdot \dot{\boldsymbol{\theta}}(t)+\boldsymbol{\Omega}^{2} \cdot \boldsymbol{\theta}(t)+\mathbf{B}_{\mathrm{inn}} \cdot\left\{\left(\bar{k}_{\mathrm{inn}}(\omega)-K_{0}\right) \boldsymbol{\theta}(t)\right\} \\
& =\mathbf{X}^{\boldsymbol{\top}} \cdot \mathbf{F}(t),
\end{aligned}
$$

once the $m \times m$ modal matrices of viscous damping, $\boldsymbol{\Xi}=\mathbf{X}^{\top} \cdot \mathbf{C}_{\mathrm{inn}} \cdot \mathbf{X}$, and rigidity influence of the inner layer on the modal subspace, $\mathbf{B}_{\mathrm{inn}}=\mathbf{X}^{\top} \cdot \mathbf{L}_{\mathrm{inn}} \cdot \mathbf{X}$, have been introduced.

When compared to the modal equations of motion of the undamped system (Eq. (23)), the most striking difference in Eq. (29) is the presence of the mixed time-frequency term $\mathbf{B}_{\text {inn }} \cdot\left\{\left(\bar{k}_{\text {inn }}(\omega)-K_{0}\right) \boldsymbol{\theta}(t)\right\}$ which is related to the rate-dependent part of the reaction forces experienced by the viscoelastic inner layer. Looking now at Eqs. (2) to (5), the mixed time-frequency 
product into curly brackets turns out to be equivalent to:

$$
\left(\bar{k}_{\mathrm{inn}}(\omega)-K_{0}\right) \boldsymbol{\theta}(t)=\left(\bar{\varphi}_{\mathrm{inn}}(t)-K_{0}\right) * \boldsymbol{\theta}(t)=K_{1} \boldsymbol{\lambda}_{1}(t)
$$

where $\boldsymbol{\lambda}_{1}(t)=\left\{\lambda_{1,1}(t) \cdots \lambda_{1, m}(t)\right\}^{\top}$ is the array of additional time-varying internal variables, each one associated with a modal coordinate. Furthermore, according to Eq. (6), the time evolution of this new array $\boldsymbol{\lambda}_{1}(t)$ is ruled by:

$$
\dot{\boldsymbol{\lambda}}_{1}(t)=\dot{\boldsymbol{\theta}}(t)-\frac{1}{\tau_{1}} \boldsymbol{\lambda}_{1}(t)
$$

in which $\tau_{1}$ is still the relaxation time of the Maxwell's element used in modelling the viscoelastic inner layer.

Finally, Eqs. (29), (30) and (31) can be arranged in a more effective state-space form:

$$
\dot{\mathbf{y}}(t)=\mathbf{D} \cdot \mathbf{y}(t)+\mathbf{G} \cdot \mathbf{F}(t),
$$

where $\mathbf{y}(t)=\left\{\boldsymbol{\theta}(t)^{\boldsymbol{\top}} \dot{\boldsymbol{\theta}}(t)^{\boldsymbol{\top}}: \boldsymbol{\lambda}_{1}(y)^{\boldsymbol{\top}}\right\}^{\boldsymbol{\top}}$, is the enlarged state array, while dynamic matrix $\mathbf{D}$ and load influence matrix $\mathbf{G}$ are so defined:

$$
\mathbf{D}=\left[\begin{array}{c:c:c}
\mathbf{O}_{m \times m} & \mathbf{I}_{m} & \mathbf{O}_{m \times m} \\
\hdashline-\mathbf{\Omega}^{2} & -\mathbf{\Xi} & -K_{1} \mathbf{B}_{\mathrm{inn}} \\
\hdashline \mathbf{O}_{m \times m} & \mathbf{I}_{m} & -\frac{1}{\tau_{1}} \mathbf{I}_{m}
\end{array}\right] ; \quad \mathbf{G}=\left[\begin{array}{c}
\mathbf{O}_{m \times m} \\
\hdashline \mathbf{X}^{\mathbf{\top}} \\
\hdashline \mathbf{O}_{m \times m}
\end{array}\right]
$$

in which $\mathbf{I}_{s}$ is the identity matrix of size $s$ and $\mathbf{O}_{r \times s}$ stands for a zero matrix with $r$ rows and $s$ columns.

From a mathematical point of view, Eq. (32) constitutes a set of inhomogeneous linear differential equations with constant coefficients, whose solution can be sought with any standard technique. This mathematical form, very convenient from a computational point of view, is possible in our formulation because the viscoelastic properties of the inner layer are factored into a frequency factor, $\bar{k}_{\mathrm{inn}}(\omega)$, and a coordinate factor $\alpha_{\mathrm{K}}(z)$. Interestingly, Eqs. (29) 
and (31) are coupled just by the modal matrices $\boldsymbol{\Xi}$ and $\mathbf{B}_{\text {inn. }}$. When these matrices are diagonal, or when their out-of-diagonal terms are negligible, the dynamic system becomes classically damped, in the sense that the modes of vibration are decoupled. Moreover, as pointed out in previous works dealing with tall buildings [22] and railway tracks [23], modal stiffness and damping in this case are characterized by modal relaxation functions, which can be easily defined starting from the knowledge of the relaxation function of the viscoelastic components.

\section{Numerical applications}

\subsection{Modal shapes and modal frequencies}

For the purposes of numerical validation, the proposed procedure is initially applied to evaluate natural frequencies and modal shapes of three undamped double-beam systems, with different mechanical parameters and all having $\zeta_{1}=\zeta_{2}=0$ and $K_{1}=0$.

In a first stage, the variant V1 considered by Oniszczuk in Ref. [12] is studied. In this example, both outer beams are homogeneous and simply supported at their ends. The length is $L=10 \mathrm{~m}$, the core is assumed to be massless, i.e. $\mu_{\text {inn }}(z)=0$, while the mechanical parameters of the top beam are: $\rho_{1}=2,000 \mathrm{~kg} / \mathrm{m}^{3}, E_{1}=10 \mathrm{GPa}, A_{1}(z)=500 \mathrm{~cm}^{2}$ and $I_{1}(z)=40,000 \mathrm{~cm}^{4}$. Mass per unit length and flexural stiffness of the bottom beam are $\mu_{2}(z)=\mu_{1}(z) / 2=50 \mathrm{~kg} / \mathrm{m}$ and $\kappa_{2}(z)=\kappa_{1}(z) / 2=2,000 \mathrm{kN} / \mathrm{m}^{2}$, respectively: i.e. the bottom beam is lighter and more flexible. The stiffness of the elastic inner layer is $K_{0}=200 \mathrm{kN} / \mathrm{m}^{2}$.

Fig. 2 shows the first six modal shapes $(m=6) \tilde{\mathbf{v}}_{j}(z)$, given by Eq. (21), 


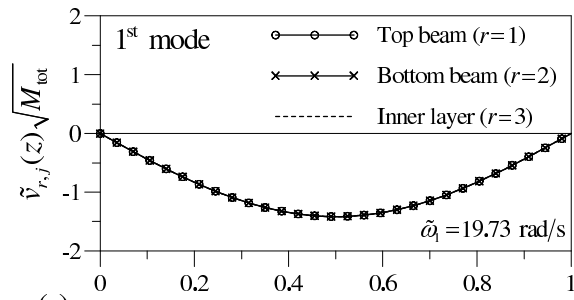

(a)

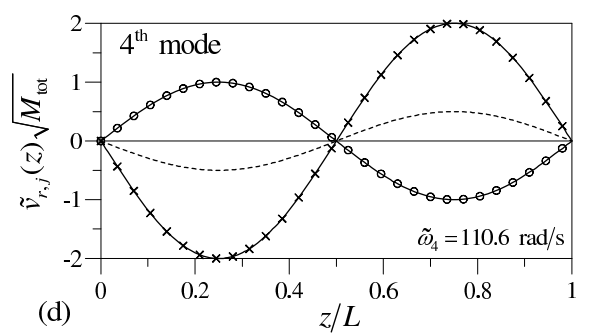

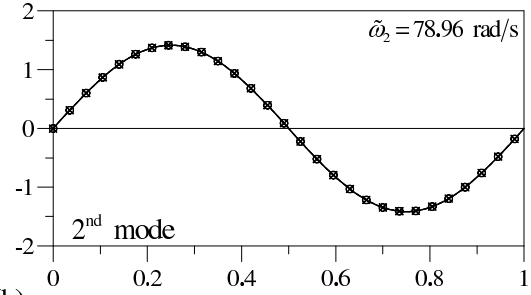

(b)

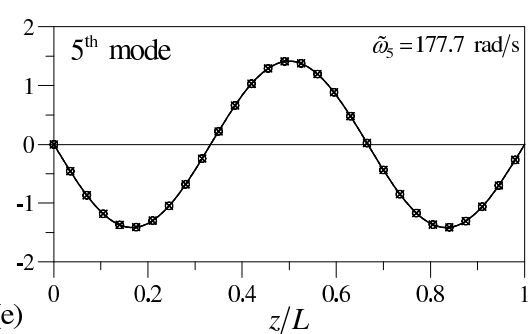

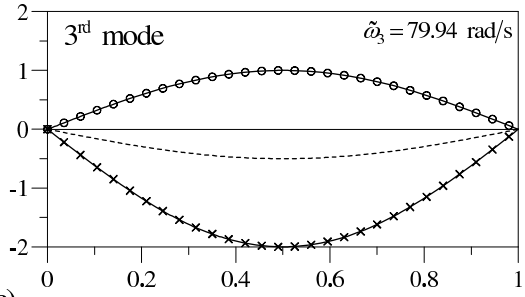

(c)

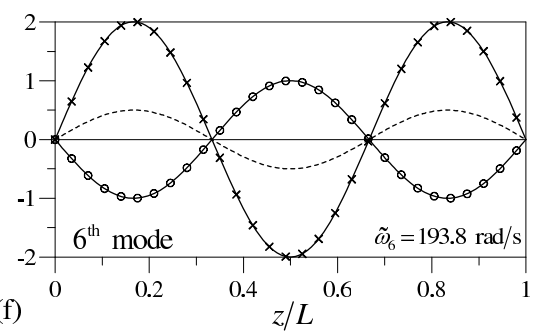

Figure 2: First six modal shapes and natural circular frequencies evaluated for variant V1 of the double-beam system considered in Ref. [12]

along with the corresponding natural circular frequencies $\tilde{\omega}_{j}$. These results are obtained with six assumed modes for each layer $(n=6)$. It is worth noting that the natural circular frequencies so computed are in perfect agreement with the exact values reported in Ref. [12], as in this example the sinusoidal assumed modes the three layers match perfectly with the exact modes of vibration of the combined system. Furthermore, as analytically predicted therein, first, second and fifth modal shapes are characterised by synchronous vibrations of the outer beams, so that the inner layer is not deformed: as a consequence, $\tilde{\omega_{1}}, \tilde{\omega}_{2}$ and $\tilde{\omega}_{5}$ do not depend on the stiffness $K_{0}$.

In a second stage, variant V2 of double-beam systems reported in Ref. [12] is considered. In this case, the top beam is the same as in variant V1 previously examined, while the bottom beam has same mass per unit length, $\mu_{2}(z)=\mu_{1}(z)=100 \mathrm{~kg} / \mathrm{m}$ and double the flexural rigidity of the top 


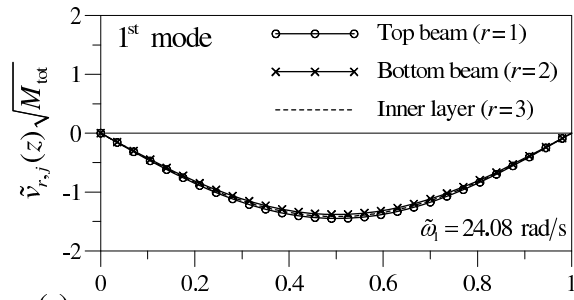

(a)

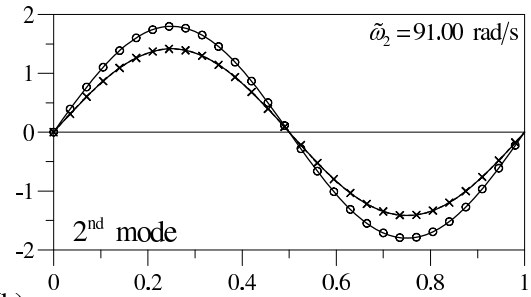

(b)

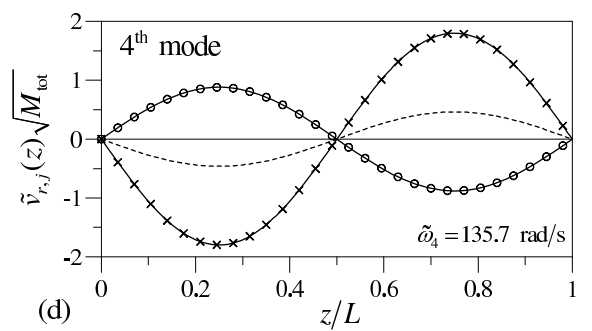

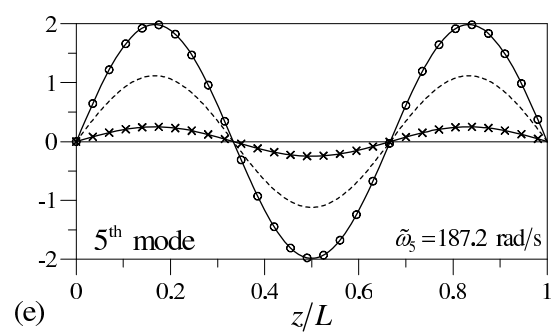

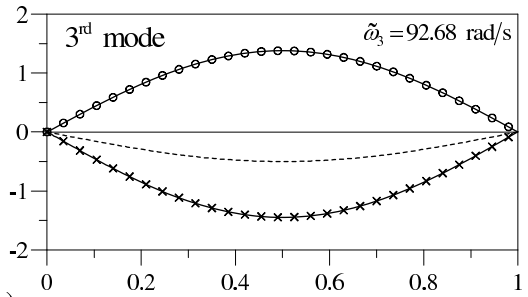

(c)

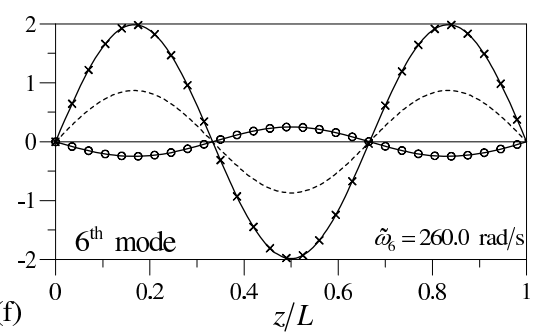

Figure 3: First six modal shapes and natural circular frequencies evaluated for variant V2 of the double-beam system considered in Ref. [12]

beam, $\kappa_{2}(z)=2 \kappa_{1}(z)=8,000 \mathrm{kN} / \mathrm{m}^{2}$. The stiffness of the inner layer is $K_{0}=400 \mathrm{kN} / \mathrm{m}^{2}$.

Fig. 3 shows the first six modal shapes and the associated natural circular frequencies $(m=6)$, as evaluated by using six assumed modes for each layer $(n=6)$. Also in this case the results of the proposed approach are in good agreement with the closed-form expressions provided in Ref. [12]. Interestingly, the inner layer is transversally deformed in all the modal shapes of this variant, and therefore all the natural frequencies depend on the stiffness $K_{0}$. It is worth mentioning that very similar results have been presented in the Ref. [20] for the same variants V1 and V2, in which however a more complicated and time-consuming procedure was adopted.

In a third stage, aimed at showing the capacity of the proposed approach to deal with double-nanobeam systems (e.g. Refs. $[7,6]$ ), the in-phase 


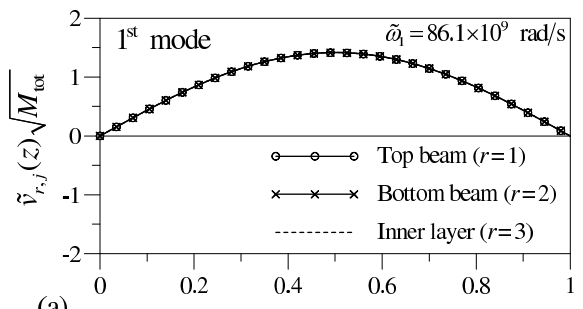

(a)

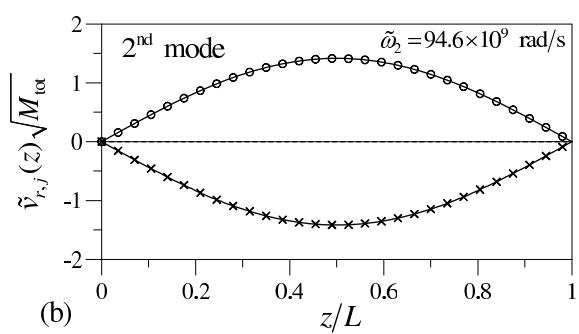

Figure 4: First in-phase (a) and out-of-phase (b) modal shapes and natural circular frequencies evaluated for the double-nanobeam system considered in Ref. [7]

and out-of-phase modal shapes and natural frequencies of a coupled pair of carbon nanotubes have been computed. The mechanical properties of outer nanobeams and inner elastic medium are: $L=20 \mathrm{~nm}, \rho_{1}=\rho_{2}=$ $2,300 \mathrm{~kg} / \mathrm{m}^{2}, E_{1}=E_{2}=971 \mathrm{GPa}, A_{1}(z)=A_{2}(z)=\pi R^{2}, I_{1}(z)=I_{2}(z)=$ $\frac{\pi}{4} R^{4}, R=0.34 \mathrm{~nm}$ being the radius of the nanotubes, and $K_{0}=10 E_{1} I_{1} / L^{4}$. Fig. 4 reports the modal properties for the first two modes of vibration ( $m=2$ ) as obtained with $n=4$ assumed mode for each layer. The same results can be recovered as a particular case by neglecting the non-local effects in the formulation proposed in Ref. [7].

\subsection{Forced vibrations}

Following the modal analyses reported in the previous subsection, validating the proposed approach against results already available in the literature for undamped double-beam systems and homogeneous distributions of mass 


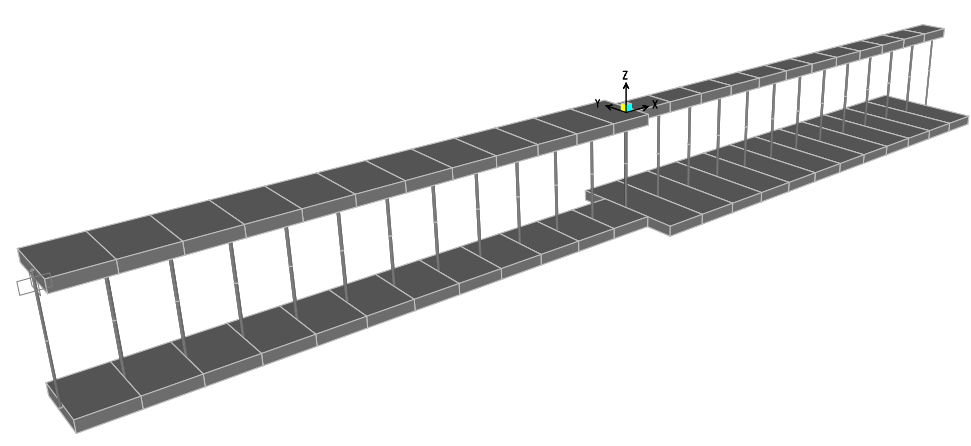

Figure 5: Elastic finite-element model built with SAP2000 [38]

and stiffness, our numerical applications proceed with forced vibration analyses of double-beam systems with both viscous (outer layers) and viscoelastic (inner layer) damping and inhomogeneous inertia and rigidity.

The mechanical parameters of the objective double-beam system are as follows: length $L=10 \mathrm{~m}$; Young's modulus $E_{r}=10 \mathrm{GPa}$, mass density $\rho_{r}=2,000 \mathrm{~kg} / \mathrm{m}^{3}$ and viscous damping ratio $\zeta_{r}=0.05$ for both outer beams $(r=1,2)$; mass per unit length $\bar{m}_{\text {inn }}=12 \mathrm{~kg} / \mathrm{m}$, Winkler-type equilibrium modulus $K_{0}=30 \mathrm{kN} / \mathrm{m}^{2}$, and Maxwell's parameters $K_{1}=5 K_{0}$ and $\tau_{1}=$ $0.2 \mathrm{~s}$ for the inner layer (see Fig. 1a). The boundary conditions are ClampedFree for the top beam and Free-Clamped for the bottom beam.

Stepped geometries are assumed for the three layers, all experiencing a sudden variation of mass and stiffness at midpspan position $(z=L / 2)$, while taking constant values in each half of the structure. Fig. 5 shows the finiteelement model of the objective double-beam system built in SAP2000 [38] with 48 Euler-Bernoulli beam elements (outer beams), 50 uniaxial bar elements (inner layer), 150 nodes and 296 degrees of freedom. This model is used herein to validate the modal properties delivered by the proposed Galerkin- 
type discretisation of the equations of motion in presence of inhomogeneous distributions of mass and stiffness.

Mathematical expressions of cross-sectional area and second moment of outer beams are:

$$
\begin{gathered}
A_{1}(z)=A_{\text {ref }}[1-0.5 \mathcal{U}(z-L / 2)] \\
I_{1}(z)=I_{\text {ref }}[1-0.5 \mathcal{U}(z-L / 2)] ; \\
A_{2}(z)=A_{\text {ref }}[1+\mathcal{U}(z-L / 2)] ; \\
I_{2}(z)=I_{\text {ref }}[1+\mathcal{U}(z-L / 2)]
\end{gathered}
$$

$A_{\text {ref }}=600 \mathrm{~cm}^{2}$ and $I_{\text {ref }}=5,000 \mathrm{~cm}^{4}$ being the reference values at the left-hand end of outer beams $(z=0)$, while $\mathcal{U}$ is the Heaviside's unit step function, defined in sub-section 2.1. The dimensionless influence functions for the inner layer are stepped as well:

$$
\alpha_{\mathrm{M}}(z)=\alpha_{\mathrm{K}}(z)=1-0.5 \mathcal{U}(z-L / 2)
$$

Fig. 6 displays the first nine pairs of modal shapes and undamped natural circular frequencies $(m=9)$ of the objective double-beam system, as evaluated with the proposed approach by considering nine assumed modes for each layer $(n=9)$. Interestingly, in the first six modes (top two rows in Fig. 6) the deformed shape of the inner layer (dashed line) always passes through nodal points where those of the outer beams cross each other, and therefore in this case outer beams' deflections are sufficient to represent core's deformations (e.g., as in the procedure proposed in Ref. [20]). The deformed shapes of the inner layer become more complicated in higher modes of vibration (bottom row in Fig. 6), as they do not always pass through the nodal points (see 


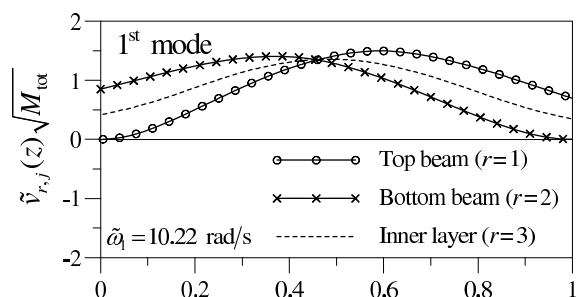

(a)

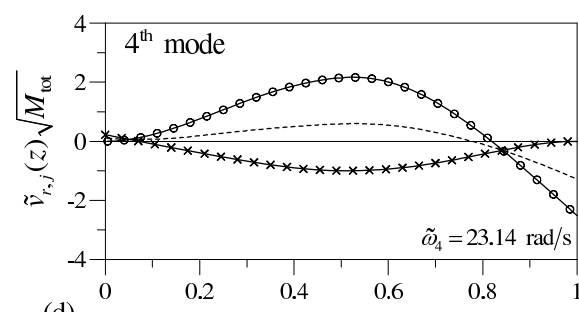

(d)

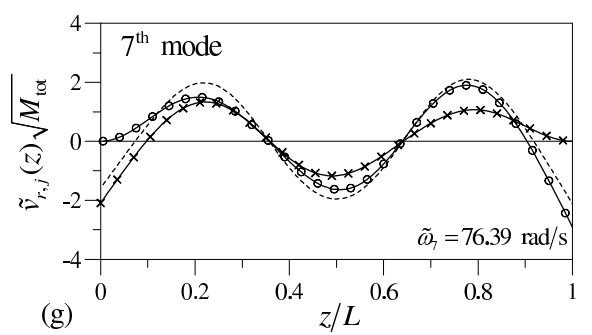

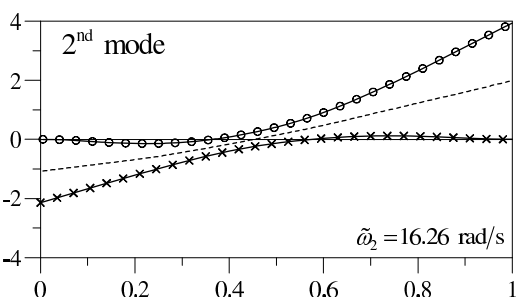

(b)

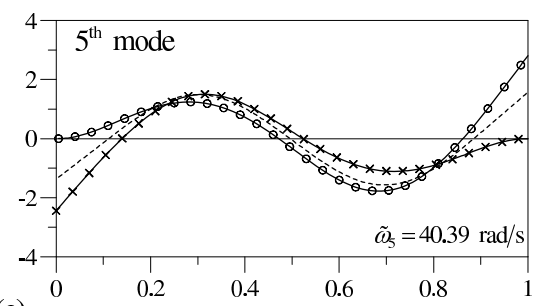

(e)

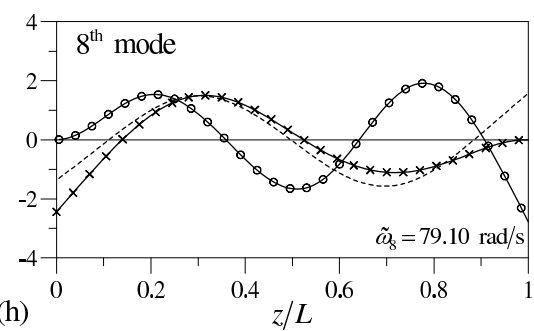

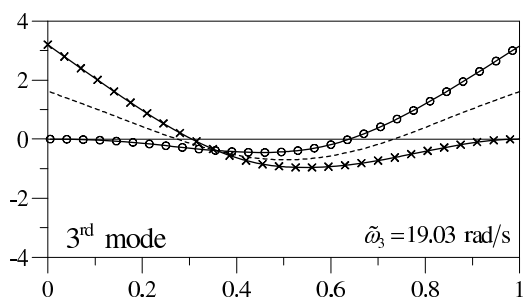

(c)

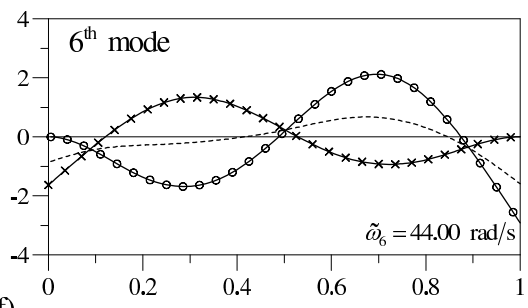

(f)

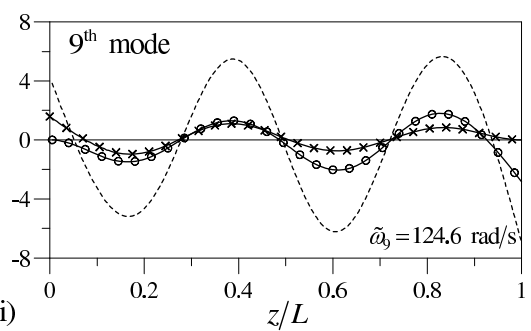

Figure 6: First nine modal shapes and undamped natural circular frequencies of the double-beam system considered in sub-section 5.2 


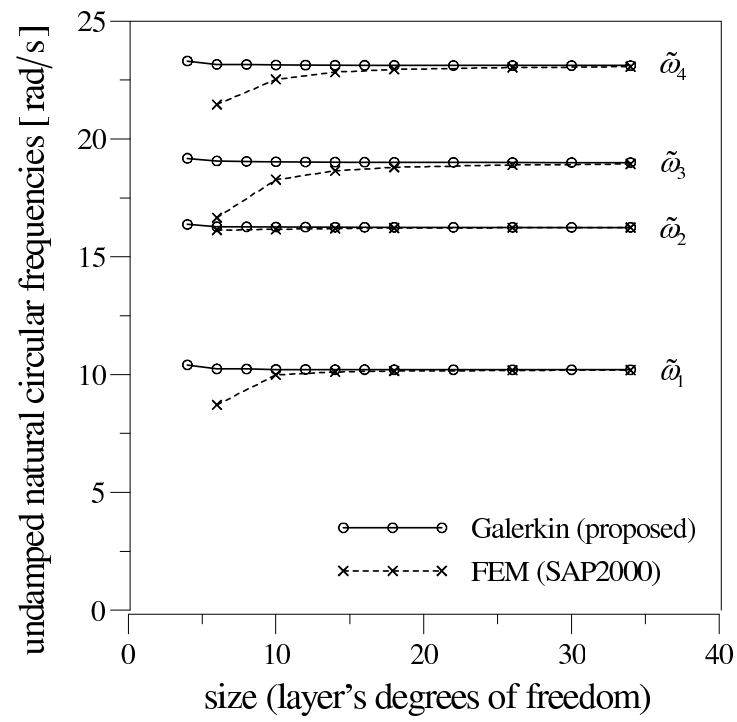

Figure 7: Convergence study for the first four natural circular frequencies

right-hand side of 7th and 8th mode) and may have larger amplitude (see 9th mode).

Fig. 7 compares the convergence rate for proposed Galerkin-type approach (denoted with circles) and standard finite element method (FEM, denoted with crosses). It appears that both techniques converge to the same values of undamped natural circular frequencies for the first four modes of vibration, although the proposed approach is faster: that is, when 10 degrees of freedom (i.e. 5 translations and 5 rotations) are considered per each layer, the inaccuracy of the FEM modelling can be as large as $12 \%$ for the first mode and $9 \%$ for the third mode; on the contrary, the inaccuracy of the proposed Galerkin-type modelling with just 8 assumed modes per layer does not exceed $0.1 \%$ for all the four modes, which makes this approach preferable from a computational point of view. 
Aimed at studying the forced vibration of the objective double-beam system, a uniform dynamic load is considered to be applied on the righthand side of the top beam, while bottom beam and inner layer are not forced. Accordingly, the array of generalised forces (see Eq. (18b)) can be expressed as:

$$
\mathbf{F}(t)=\overline{\mathbf{F}} w(t)
$$

where $w(t)$ is the time-varying scalar force per unit lenght, while $\overline{\mathbf{F}}$ is the $(3 n) \times 1$ spatial influence array, given by:

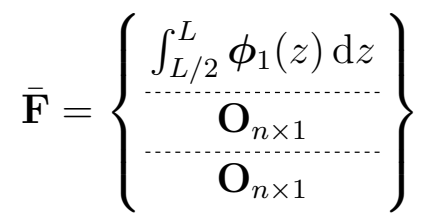

In a first stage, a frequency-domain approach is pursued. To do this, Fourier's transform of both sides of Eq. (32) are taken:

$$
\mathcal{F}\langle\mathbf{y}(t)\rangle=\mathbf{H}(\omega) \cdot \mathcal{F}\langle\mathbf{F}(t)\rangle
$$

where $\mathbf{H}(\omega)$ is the $(3 m) \times(3 n)$ complex-valued matrix collecting the FRFs (frequency response functions) of the state variables listed in the three $n$ dimensional arrays $\boldsymbol{\theta}(t), \dot{\boldsymbol{\theta}}(t)$ and $\boldsymbol{\lambda}_{1}(t)$, which in turn is so defined:

$$
\mathbf{H}(\omega)=\left[\imath \omega \mathbf{I}_{3 m}-\mathbf{D}\right]^{-1} \cdot \mathbf{G} .
$$

Recalling now Eqs. (8), (9) and (22), the FRFs of transverse displacements at a given abscissa $z=\bar{z}$ for the selected load pattern can be expressed as:

$$
\mathcal{F}\left\langle\left\{\begin{array}{c}
v_{1}(\bar{z}, t) \\
v_{2}(\bar{z}, t) \\
v_{3}(\bar{z}, t)
\end{array}\right\}\right\rangle=\left\{\begin{array}{r}
\bar{Z}_{1}(\omega) \\
\bar{Z}_{2}(\omega) \\
\bar{Z}_{3}(\omega)
\end{array}\right\} \mathcal{F}\langle w(t)\rangle=
$$




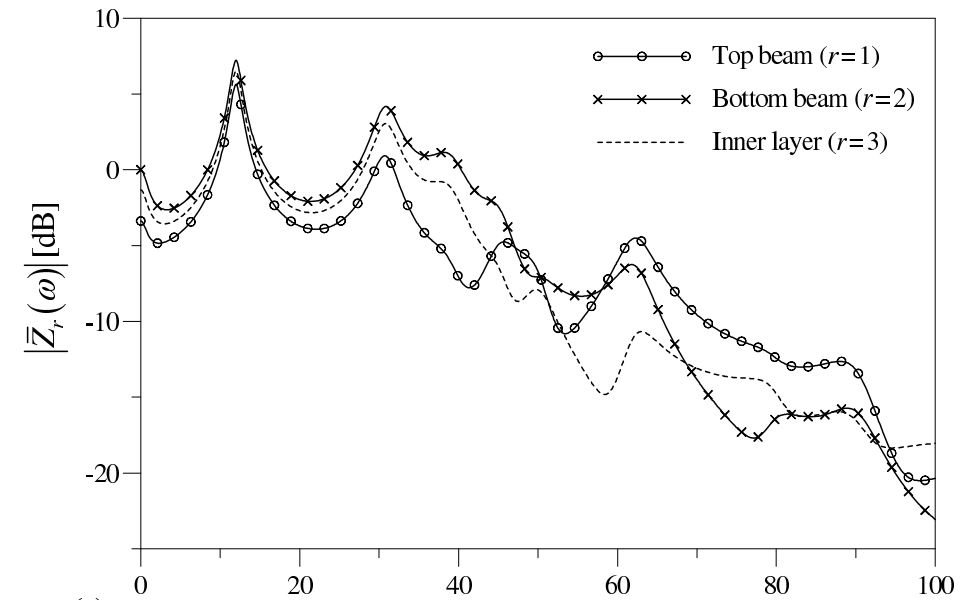

(a)

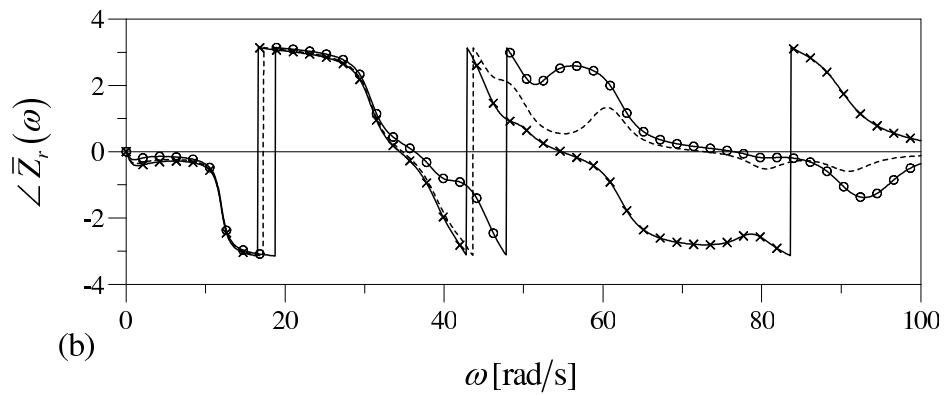

Figure 8: Modulus (a) and phase (b) of the complex-valued frequency response functions of transverse displacements at $z=L / 6$ 
Absolute value $\left|\bar{Z}_{r}(\omega)\right|\left(\right.$ in $\mathrm{dB}$ ) and phase $\angle \bar{Z}_{r}(\omega)$ (in rad) are plotted in Fig. 8 for the three layers of the double-beam system under investigation at the abscissa $\bar{z}=L / 6$. It can be observed that in the low-frequency range $(\omega<40 \mathrm{rad} / \mathrm{s})$, the absolute value for the inner layer (dashed line) always falls between those of the outer beams (circles and crosses), therefore core's deformation is only dictated by outer beams' deflections. This well-ordered behaviour vanishes in the high-frequency range $(\omega>50 \mathrm{rad} / \mathrm{s})$, where the absolute value for the inner layer shows a much more complicated pattern. The regular nature of vibrations at low frequencies is confirmed by the phase plots for the three layers (Fig. 8(b)), which are very close each other for $\omega<40 \mathrm{rad} / \mathrm{s}$, and separate for $\omega>50 \mathrm{rad} / \mathrm{s}$.

It is also worth noting than the absolute values of the three FRFs $\left|\bar{Z}_{r}(\omega)\right|$ in Fig. 8(a) show a relative minimum in the interval $\left[0, \tilde{\omega}_{1}\right]$, corresponding to the quasi-static frequency range. This is due to the relaxation processes within the viscoelastic core, which cannot be represented with a simpler viscous damping model, therefore confirming the need of a more accurate modelling for composite double-beam systems with viscoelastic core.

In a second stage, the dynamic response is sought in the time domain. The excitation is chosen as superposition of low-frequency sine and highfrequency sweep functions:

$$
w(t)=1 \mathrm{kN} / \mathrm{m} \times\left[\sin \left(\frac{2 \pi t}{T_{\mathrm{f}}}\right)+\sin \left(\frac{\Omega_{\mathrm{f}} t}{3}+\frac{2 \Omega_{\mathrm{f}} t^{2}}{3 T_{\mathrm{f}}}\right)\right],
$$

in which $T_{\mathrm{f}}=12 \mathrm{~s}$ and $\Omega_{\mathrm{f}}=15 \pi \mathrm{rad} / \mathrm{s}$.

The following unconditionally-stable single-step numerical scheme of so- 


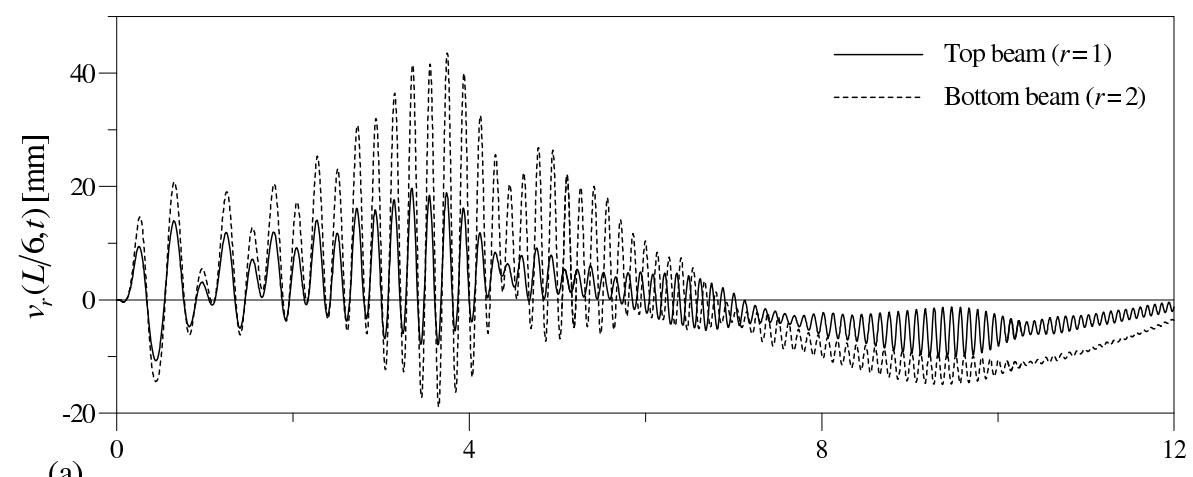

(a)

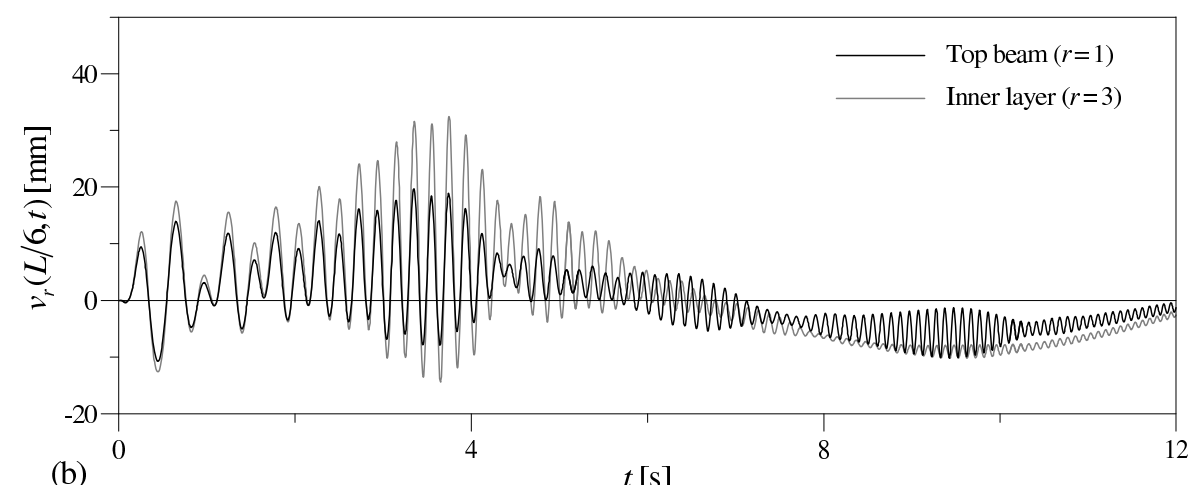

Figure 9: Time histories of transverse displacements experienced by different layers at $z=L / 6$ 
lution is adopted to solve Eq. (32):

$$
\mathbf{y}(t+\Delta t)=\mathbf{\Theta}(\Delta t) \cdot \mathbf{y}(t)+\boldsymbol{\psi}_{0}(\Delta t) w(t)+\boldsymbol{\psi}_{1}(\Delta t) w(t+\Delta t),
$$

where the transition matrix is given by:

$$
\boldsymbol{\Theta}(\Delta t)=\exp [\mathbf{D} \Delta t]
$$

in which the dynamic matrix $\mathbf{D}$ is defined by the first of Eqs. (33) and $\Delta t=0.004013 \mathrm{~s}$ is the selected time step, while the loading vectors take the expressions:

$$
\begin{gathered}
\boldsymbol{\psi}_{0}(\Delta t)=\left[\boldsymbol{\Theta}(\Delta t)-\frac{1}{\Delta t} \boldsymbol{\Lambda}(\Delta t)\right] \cdot \mathbf{D}^{-1} \cdot \mathbf{G} \cdot \overline{\mathbf{F}} \\
\boldsymbol{\psi}_{1}(\Delta t)=\left[\frac{1}{\Delta t} \boldsymbol{\Lambda}(\Delta t)-\mathbf{I}_{3 m}\right] \cdot \mathbf{D}^{-1} \cdot \mathbf{G} \cdot \overline{\mathbf{F}}
\end{gathered}
$$

where $\boldsymbol{\Lambda}(\Delta t)=\left[\boldsymbol{\Theta}(\Delta t)-\mathbf{I}_{3 m}\right] \cdot \mathbf{D}^{-1}$.

Previous investigations [20,39] have demonstrated stability and accuracy of the proposed scheme of numerical integration for viscoelastically damped structures. This technique is used herein to evaluate the time histories of transverse deflections experienced by outer beams and inner layer at the same location $(\bar{z}=L / 6)$ in the time interval $\left[0, T_{\mathrm{f}}\right]$. Comparisons reported in Fig. 9 reveal the quite complicated dynamics of the objective double-beam system, with a strong frequency-dependent behaviour. For instance, the top beam (solid black line) oscillates less than bottom beam (dashed line, Fig. 9(a)) and inner layer (gray line, Fig. 9(b)) when the frequency of vibration is relatively low (first half of time histories); the opposite happens when the frequency of vibration increases and the amplitude of the motion reduces drastically for bottom beam and inner layer (second half of time histories). 


\section{Conclusions}

A general method has been presented for studying transverse vibrations of a double-beam system, made of two parallel Euler-Bernoulli elastic beams continuously connected by a Winkler-type viscoelastic layer. As opposite to other techniques available in the literature (e.g. Refs. $[12,13,14]$ ), the proposed method can be used also in the general case of inhomogeneous systems and different boundary conditions; furthermore, the constitutive law adopted for the inner layer incorporates a Maxwell's element, able to describe the rate-dependent behaviour of many viscoelastic materials.

In a first stage, the kinematics of the structure has been represented through a Galerkin-type approach, requiring three sets of assumed modes for top beam, bottom beam and inner layer. These assumed modes have been conveniently selected as the first $n$ buckling modes of each layer with homogenised mechanical properties and its own boundary conditions, which in general vary from layer to layer. As such, layers' assumed modes are known in closed form and involve simple harmonic functions (and possibly constant and linear functions if the individual layer is not kinematically stable by itself).

In a second stage, the Lagrange's equations of motion have been derived for undamped double-beam systems, and then arranged in a compact state-space form, in which mass and stiffness matrices can be easily obtained through simple numerical integrations. It has been also shown that the proposed Galerkin-type approach converges faster that a classical finite-element modelling.

In a third stage, working in a reduced modal space (of dimensions $m \leq 3 n$ ), 
two different sources of damping have been embedded in the proposed modelling, namely a Rayleigh-type viscous damping for the outer beams and a Maxwell-type viscoelastic constitutive law for the core, therefore addressing the very general case of non-viscous non-proportional damping. To do so, a set of additional internal variables has been appended to the classical state variables (i.e. Lagrangian displacements and velocities), which take into account the rate-dependent rheology of the inner layer.

The numerical applications herein included demonstrate that the proposed method is accurate and versatile, being effective in both frequencyand time-domain analyses.

\section{Appendix A. Mass and stiffness coefficients}

Aim of this appendix is to provide the analytical expressions to evaluate mass and stiffness coefficients, which are introduced in Eqs. (11) and (12) and are collected in the $n \times n$ block matrices $\mathbf{M}^{(r, r)}, \mathbf{K}^{(r, s)}$ and $\Delta \mathbf{K}^{(r, r)}$ in Eqs. (19) and (25).

The generic mass coefficient $M_{j, k}^{(r, r)}$ for the $r$ th subsystem is given by:

$$
M_{j, k}^{(r, r)}=\int_{0}^{L} \mu_{r}(z) \phi_{r, j}(z) \phi_{r, k}(z) \mathrm{d} z
$$

where the mass per unit length $\mu_{r}(z)$ takes different expressions for outer beams $(r=1,2)$ and inner layer $(r=3)$, as shown in Section 3.1.

The stiffness coefficients associated with the flexural rigidity of the outer beams $(r=1,2)$ are given by:

$$
K_{i, k}^{(r, r)}=E_{r} \int_{0}^{L} I_{r}(z) \phi_{r, j}^{\prime \prime}(z) \phi_{r, k}^{\prime \prime}(z) \mathrm{d} z
$$


in which the second derivative of the generic assumed mode, $\phi_{r, j}^{\prime \prime}(z)$, is always known in closed form, being either a simple trigonometric function of the abscissa $z$ or even zero for the rigid-body modes of kinematically unstable layers.

The generic coefficient $\Delta K_{j, k}^{(r, r)}$, which take into account the additional stiffness coupling $j$ th and $k$ th assumed modes of the $r$ th outer beam due to the inner layer, can be evaluated as:

$$
\Delta K_{j, k}^{(r, r)}=2 K_{0} \int_{0}^{L} \alpha_{\mathrm{K}}(z) \phi_{r, j}(z) \phi_{r, k}(z) \mathrm{d} z .
$$

The direct stiffness coefficients $K_{j, k}^{(3,3)}$ for the inner layer are given by:

$$
K_{j, k}^{(3,3)}=4 K_{0} \int_{0}^{L} \alpha_{\mathrm{K}}(z) \phi_{3, j}(z) \phi_{3, k}(z) \mathrm{d} z .
$$

Finally, the stiffness coefficient $K_{j, k}^{(r, 3)}$, coupling the $j$ th assumed mode of the $r$ th outer beam $(r=1,2)$ and the $k$ th assumed mode of the inner layer, takes the expression:

$$
K_{j, k}^{(r, 3)}=-2 K_{0} \int_{0}^{L} \alpha_{\mathrm{K}}(z) \phi_{r, j}(z) \phi_{3, k}(z) \mathrm{d} z .
$$

It is worth noting that for homogeneous double-beam systems, i.e. when inertia and rigidity of the components do not vary with the abscissa $z$, all the above coefficients can be evaluated in closed form, without any numerical integration.

\section{References}

[1] J. Hohe, L. Librescu, Advances in the structural modeling of elastic sandwich panels, Mechanics of Advanced Materials and Structures 11 (2004) 395-424. 
[2] A. Palmeri, A state-space viscoelastic model of double-beam systems toward the dynamic analysis of wind turbine blades, in: Earth \& Space 2010 Conference, ASCE, Honolulu, 2010, published on CD-Rom.

[3] M. Eichenfield, R. Camacho, J. Chan, K. J. Vahala, O. Painter, A picogram- and nanometre-scale photonic-crystal optomechanical cavity, Nature 459 (7246) (2009) 550-U79.

[4] Q. Quan, P. B. Deotare, M. Loncar, Photonic crystal nanobeam cavity strongly coupled to the feeding waveguide, Applied Physics Letters $96(20)$.

[5] I. W. Frank, P. B. Deotare, M. W. McCutcheon, M. Loncar, Programmable photonic crystal nanobeam cavities, Optics Express 18 (8) (2010) 8705-8712.

[6] T. Murmu, S. Adhikari, Nonlocal effects in the longitudinal vibration of double-nanorod systems, Physica E: Low-dimensional Systems and Nanostructures 43 (1) (2010) 415-422.

[7] T. Murmu, S. Adhikari, Nonlocal transverse vibration of doublenanobeam-systems, Journal of Applied Physics 108 (8) (2010) 083514:19.

[8] T. Murmu, S. Adhikari, Axial instability of double-nanobeam-systems, Physics Letters A 375 (3) (2011) 601-608.

[9] A. Tadayoshi, T. Susumu, O. Norio, I. Yasuo, Vibration control of beams by beam-type dynamic vibration absorbers, ASCE Journal of Engineering Mechanics 118 (1992) 248-258. 
[10] M. F. M. Hussein, H. E. M. Hunt, Modelling of floating-slab track with continuous slab under oscillating moving loads, Journal of Sound and Vibration 297 (2006) 37-54.

[11] H. V. Vu, A. M. Ordez, B. H. Karnopp, Vibration of a double-beam system, Journal of Sound and Vibration 229 (2000) 807-822.

[12] Z. Oniszczuk, Free transverse vibrations of elastically connected simply supported double-beam system, Journal of Sound and Vibration 232 (2000) 387-403.

[13] Z. Oniszczuk, Forced transverse vibrations of an elastically connected complex simply supported double-beam system, Journal of Sound and Vibration 264 (2003) 273-286.

[14] M. Abu-Hilal, Dynamic response of a double Euler-Bernoulli beam to a moving constant load, Journal of Sound and Vibration 297 (2006) $477-491$.

[15] H. T. Banks, D. J. Inman, On damping mechanisms in beams, Journal of Applied Mechanics, ASME 58 (1991) 716-723.

[16] F. Corts, M. J. Elejabarrieta, Longitudinal vibration of a damped rod. Part I: Complex natural frequencies and mode shapes, International Journal of Mechanical Sciences 48 (9) (2006) 969 - 975.

[17] F. Corts, M. J. Elejabarrieta, Forced response of a viscoelastically damped rod using the superposition of modal contribution functions, Journal of Sound and Vibration 315 (1-2) (2008) 58 - 64. 
[18] W.-R. Chen, Bending vibration of axially loaded Timoshenko beams with locally distributed Kelvin-Voigt damping, Journal of Sound and Vibration 330 (13) (2011) 3040 - 3056.

[19] B. P. Yadav, Vibration damping using four-layer sandwich, Journal of Sound and Vibration 317 (3-5) (2008) 576 - 590.

[20] A. Palmeri, G. Muscolino, Transverse vibration of double-beam systems with viscoelastic inner layer, in: Eurodyn, Southamton, 2008, published on CD-Rom.

[21] F. J. Lockett, Nonlinear Viscoelastic Solids, Academic Press, London, 1972.

[22] A. Palmeri, F. Ricciarelli, G. Muscolino, A. De Luca, Effects of viscoelastic memory on the buffeting response of tall buildings, Wind and Structures 7 (2004) 89-106.

[23] G. Muscolino, A. Palmeri, Response of beams resting on viscoelastically damped foundation to moving oscillators, International Journal of Solids and Structures 44 (2007) 1317-1336.

[24] A. Palmeri, F. Ricciarelli, A. De Luca, G. Muscolino, State space formulation for linear viscoelastic dynamic systems with memory, ASCE Journal of Engineering Mechanics 129 (2003) 715-724.

[25] S. Adhikari, Eigenrelations for non-viscously damped systems, AIAA Journal 39 (8) (2001) 1624-1630. 
[26] D. J. McTavish, P. C. Hughes, Modeling of linear viscoelastic space structures, Transactions of ASME, Journal of Vibration and Acoustics 115 (1993) 103-110.

[27] S. Adhikari, Qualitative dynamic characteristics of a non-viscously damped oscillator, Proceedings of the Royal Society of London, SeriesA 461 (2059) (2005) 2269-2288.

[28] M. I. Friswell, D. J. Inman, Reduced-order models of structures with viscoelastic components, AIAA Journal 37 (10) (1999) 1318-1325.

[29] S. Adhikari, J. Woodhouse, Quantification of non-viscous damping in discrete linear systems, Journal of Sound and Vibration 260 (3) (2003) 499-518.

[30] S. Adhikari, N. Wagner, Direct time-domain approach for exponentially damped systems, Computer and Structures 82 (29-30) (2004) 2453-2461.

[31] A. Muravyov, Analytical solutions in the time domain for vibration problems of discrete viscoelastic systems, Journal of Sound and Vibration 199 (2) (1997) 337-348.

[32] A. Muravyov, Forced vibration responses of a viscoelastic structure, Journal of Sound and Vibration 218 (5) (1998) 892-907.

[33] S. Adhikari, N. Wagner, Analysis of asymmetric non-viscously damped linear dynamic systems, Transactions of ASME, Journal of Applied Mechanics 70 (6) (2003) 885-893. 
[34] S. Adhikari, M. I. Friswell, Y. Lei, Modal analysis of nonviscously damped beams, Journal of Applied Mechanics, ASME 74 (2007) 10261030.

[35] S. P. Timoshenko, J. M. Gere, Theory of Elastic Stability, McGraw-Hill, New York, 1961.

[36] R. Clough, J. Penzien, Dynamics of Structures, 2nd Edition, Computers and Structures, Berkeley, 1975.

[37] A. K. Chopra, Dynamics of Structures, 3rd Edition, Pearson Prentice Hall, Upper Saddle River, 2007.

[38] Computers \& Structures, Inc., SAP2000 v. 14, Computer Programme (2011).

[39] A. Palmeri, Trends in Civil and Structural Engineering Computing, Saxe-Coburg Publications, 2009, Ch. Toward overcoming the concept of effective stiffness and damping in the analysis and design of viscoelastically damped structures, pp. 267-92. 


\section{List of Figures}

1 Double-beam dynamic system (a); Standard Linear Solid (SLS) rheological model $(\mathrm{b}) \ldots \ldots \ldots \ldots$

2 First six modal shapes and natural circular frequencies evaluated for variant V1 of the double-beam system considered in Ref. $[12] \ldots \ldots \ldots \ldots \ldots \ldots$

3 First six modal shapes and natural circular frequencies evaluated for variant V2 of the double-beam system considered in Ref. $[12] \ldots \ldots \ldots \ldots \ldots \ldots$

4 First in-phase (a) and out-of-phase (b) modal shapes and natural circular frequencies evaluated for the double-nanobeam system considered in Ref. [7] . . . . . . . . . . . . . . . . . . 21

$5 \quad$ Elastic finite-element model built with SAP2000 [38] . . . . 22

$6 \quad$ First nine modal shapes and undamped natural circular frequencies of the double-beam system considered in sub-section

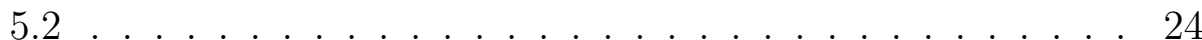

$7 \quad$ Convergence study for the first four natural circular frequencies 25

8 Modulus (a) and phase (b) of the complex-valued frequency response functions of transverse displacements at $z=L / 6$. . 27

9 Time histories of transverse displacements experienced by different layers at $z=L / 6 \ldots \ldots$. . . . . . . . . . . . 29 


\section{List of Tables}

1 Assumed modes for the outer beams (see e.g. Ref. [35]) . . . . 9

2 Additional rigid-body modes for kinematically unstable beams 10 